Review Article

\title{
Role of Long Non-coding RNAs in Sculpting Neurons
}

\author{
MAYURESH ANANT SARANGDHAR ${ }^{1,2}$, DIVYA CHAUBEY ${ }^{1,2}$ and BEENA PILLAI ${ }^{1,2 *}$ \\ ${ }^{1}$ CSIR-Institute of Genomics and Integrative Biology (IGIB), Mathura Road, New Delhi 110 025, India \\ ${ }^{2}$ Academy of Scientific and Innovative Research (AcSIR), New Delhi, India
}

(Received on 23 October 2017; Revised on 03 March 2018; Accepted on 30 March 2018)

\begin{abstract}
The morphology of neurons, a collective measure of features like the size, number, length, density and branching patterns of dendrites, as well as axonal features like length and diameter of the axon, is critical to its function. At a finer level of detail, dendrites bear membranous bulb-like projections on their surfaces called dendritic spines. Dendritic spines are the sites of neurotransmitter signalling and undergo activity dependent dynamic changes in shape associated with a regulated rearrangement of the actin cytoskeleton. Here, we provide an overview of four lncRNAs: MALAT1, BC200, 7H4 and Durga, which are implicated in altering neuronal architecture. We have recently discovered the lncRNA named Durga, in the zebrafish genome, in close proximity to Kalirin gene, which modulates expression of Kalirin and dendritogenesis. Kalirin is a Rho-GEF kinase that is involved in the cytoskeletal rearrangements that occur during dendritogenesis and dendrite spine morphologenesis. It is closely associated with proteins of post-synaptic density, a structure found at dentritic spines, and through sequential activation of other RhoGTPases, brings about cytoskeletal changes in response to developmental signals and neurotransmitters. Here, we provide an overview of dendritogenesis, the molecular basis of dendrite and dendritic spine formation and discuss the involvement of lncRNA in the process.
\end{abstract}

Keywords: Non-coding RNAs; Long Non-coding RNAs; Dendritogenesis; Dendritic Spines; Synapticplasticity; Neuronal Architecture

\section{Introduction}

Neuronsarethe basic information processing units of nervous system composed of three major structures: soma, dendrites and axons. Dendrites, the branched projections of neurons, receive and integrate synaptic inputs through small protrusions called dendritic spines. The soma subsequently processes the synaptic inputs and passes it to other neurons via elongated projections, the axons. A single axon makes synapses with dendritic spines from multiple neurons. A synapse is formed by three ultramicroscopic structural components: presynaptic terminal, postsynaptic terminal and synaptic cleft. At the presynaptic terminal on dendritic spines, neurotransmitters are packaged into synaptic vesicles and upon calcium influx released into the space of the synaptic cleft (Pitman, 1984). Receptors at postsynaptic terminals receive and interpret messages from presynaptic terminals. At the postsynaptic plasma membrane many receptors, effectors, adhesion proteins, kinases, phosphatases and cytoskeletal elements get assembled into an electron dense region called postsynaptic density (PSD) of synapse (Okabe, 2007). Compared to our knowledge of the proteins localized to such specialized compartments of neurons, we know little about the RNA species present in these regions. Messenger RNA of several proteins, notably Actin and Fmrp, are enriched and locally translated at the synapse (Bramham and Wells, 2007). Various classes of noncoding RNAs account for the bulk of eukaryotic cellular RNAs. Recently, a large number of less abundant non-coding RNAs - the long non-coding RNAs (lncRNAs), have been discovered, many of them are regulatory in function. IncRNAs are transcripts without any protein coding capability, they have now been located in every compartment of the cell, including common locations such as the nucleus and the cytoplasm; or specialized areas, like axons

*Authorfor Correspondence: E-mail: beena@igib.in 
and dendrites of neurons. Some of these lncRNAs regulate gene expression and signaling events at various stages of brain development, including neural stem cell differentiation (Wu et al., 2013; Ramos et al., 2015).

Here, we briefly review neuronal architecture and then focus on IncRNAs which reside within dendrites and axons. They may be highly enriched near synapses, modulated by neuronal electrical activity and may participate in plasticity responses, such as learning and memory. Based on the available evidence, we also point out gaps in the current understanding of IncRNA at the synapse and suggest promising lines of investigation that will help in future to elucidate the purpose of lncRNAs that are docked at such specialized compartments and their relevance in human disease.

\section{Nuts and Bolts of Neuronal Architecture}

Neurons are specialized cells of the brain involved in rapidly transmitting signals- electrical and chemical from cell to cell. Neurons receive signals from neighboring cells, integrate these inputs to determine if they will be transmitted further and finally communicate it to target cells. The target cells may be other neurons in a network or other cell types like muscle cells. Ramon y Cajal's early description of the neurons in brain sections based on the staining of the Golgi bodies is replete with references to the shapes of the cells. His beautiful and intricate drawings of neurons made by meticulously following the course of each neuron in brain sections give us our earliest insights into the complexity of cell shape and its relation to the function of the neuron (Cajal, 1906). The main cell body or soma of the neuron contains the nucleus and is also a site for the central synthesis of protein. The cell body is surrounded by protrusions of varying length and branching pattern. Typically, a single, relatively long axon and a number of shorter highly branched dendrites together specify the morphology of neuron. Besides the shape, absence of the myelin sheath and presence of tiny bulb like membranous protrusions called dendritic spines decorated on the surface differentiate dendrites from the axon. The dendritic spines are responsible for local increase in surface area and provide autonomous compartments within the neuron for local regulation (Nimchinsky et $a l .$, 2002). The shape, density and distribution of dendritic spines are critical features of neurons with strong functional implications. Dendritic spines can be thought of as sub-cellular locations containing neurotransmitter receptors, organelles and molecules involved in synaptic functions. Since each dendritic spine connects to a single excitatory axon, it is thought to be the unit of synaptic activity. The endoplasmic reticulum of the neuron may penetrate into the spine and facilitate local and strong calcium flux following neurotransmitter binding to the receptor (Kovalchuk et al., 2000).

Dendrites receive the excitatory and inhibitory signals coming into the neuron, the sum of which determines its response. A neuron uses its dendrites to sample an area around it called the dendritic field. During development, the number of dendrites increase and are subsequently pruned (Riccomagno and Kolodkin, 2015). Mature neurons usually have a stable dendrite arborization pattern. At the molecular level, a large number of proteins are implicated in the determination of dendrite numbers, density and branching patterns. Mutations in transcription factors like Abrupt and Cut and cell surface receptors like Robo show altered dendrite genesis (Jan and Jan, 2010). The small RhoGTPaseRhoA and RAC1 have opposite effects on dendrite length and branching. PSD95 a protein present in the postsynaptic density is also involved in regulation of dendritogenesis and dendrite spine morphogenesis (Penzes et al., 2011). In young hippocampal neurons, PSD95 is readily detected outside the synapses. Over-expression of PSD95 reduces secondary branching of dendrites. Besides, its role in activity independent regulation of dendrite branching, PSD95 is known to modulate dendrite spine morphology in an activity dependent manner. PSD95 is connected to the cytoskeleton, indirectly, by intermediate proteins and can act as a scaffolding protein in the dendritic spine (Hu et al., 2011). Proteins like Cypin and Kalirin 7 that interact with PSD95 are also important in dendritogenesis and dendrite spine morphogenesis (Xie et al., 2007).

\section{Diversity of Neuronal Architectures}

Neurons adopt diverse shapes and sizes depending on their specific functions. For instance, a motor neuron that connects the spinal cord to limbs can be about a meter long while most other neurons are just a few microns in length. Some model organisms, for 
instance the sea slug, Aplysia, are chosen for their gigantic neurons that are especially amenable to electrophysiological studies (Moroz, 2011). The giant axon of the squid can be upto $1 \mathrm{~mm}$ in diameter, allowing rapid transmission of signals and triggering powerful contractions of the muscular body to eject a stream of water that propels the squid away from danger. The morphology of a typical neuron with short branching dendrites surrounding the soma and long axon extending away from the cell body is found in motor neurons. Bipolar neurons may have a single basal dendrite and axon of similar length, emerging from the central cell body. Interneurons that connect between neurons may be bipolar and depending on whether they go between local or distal neurons they may have short or long axons and dendrites. True unipolar neurons found in invertebrates have a denuded appearance with the dendrites being absent and a single long axon that connects to the target cell. A pseudounipolar cell arises from a bipolar cell when the cell body is located a small distance away from the dendrite and axon. In such cells, the axon and dendrite appear to be connected directly - a morphology commonly found in sensory neurons. In pseudounipolar cells, a single process emerging from the cell body is split into equally long processes: the dendrite and the axon (Kandel et al., 2013). Thus, it is clear that the nervous system consists of morphologically diverse neurons (Fig. 1).

The shapes of neurons are directly linked to their functions (Vetter et al., 2001). Presumably, inappropriate changes in the shapes of neurons may lead to dysfunctional neural networks and disease. Until recently, the relevance of neuronal form was not studied directly in neurodegenerative diseases and altered neuronal shape was hardly explored as an underlying cause for functional deficits (Šišková et al., 2014). From studies using labeling on sections of postmortem tissue of Huntington's disease patient's brain it is now clear that an overall reduction in dendrite length and abnormal patterns of branching are seen in neurodegenerative diseases (Graveland et al., 1985). In the early stages of the disease, striatal spiny neurons show an abnormal bending of distal dendrites and increased branching. While a number of cortical, prefrontal pyramidal neurons degenerate, some pyramidal neurons may show increased dendritic spine length, density and volume (Sotrel et al., 1993). Besides Huntington's disease, dendritic morphology

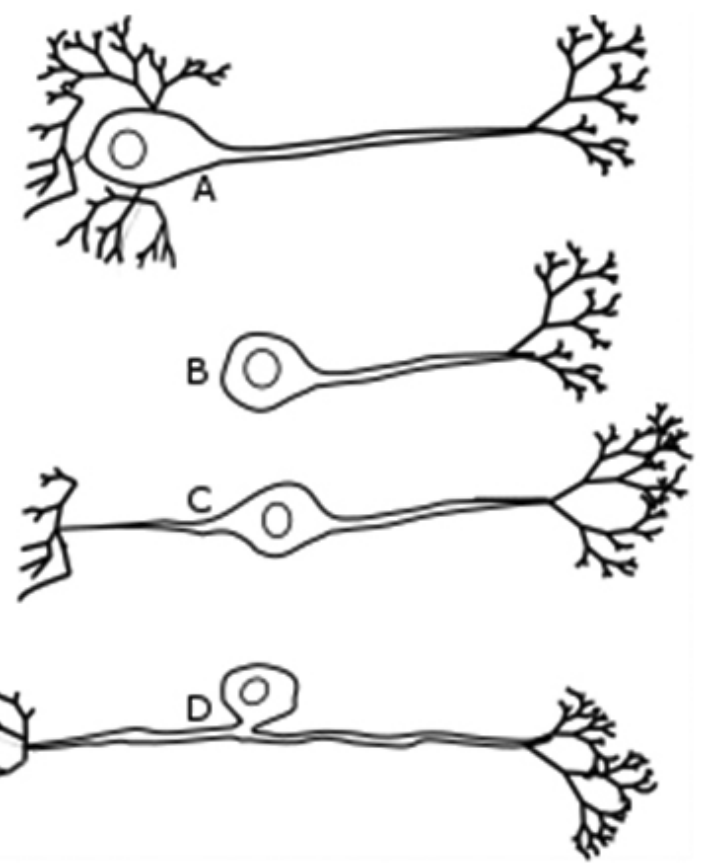

Fig. 1: Neuronal morphology varies according to function. (A) A motoneuron is typically multipolar whereas (B) invertebrates may have unipolar cells. (C) The retina contains bipolar neurons and (D) Sensory neurons are pseudo-unipolar neurons

is affected in a variety of neurodegenerative diseases of the brain including taupathies, prion diseases and alpha-synucleopathies (Kweon et al., 2017).

\section{Long Non-coding RNAs: Active Players at Dendrites and Synapses}

On the evolutionary scale, biological complexity has steadily increased, without a corresponding change in a number of protein coding genes, whereas noncoding portion of the genome has undergone substantial change. It is now a well accepted fact that in higher organisms, majority of the genome gives rise to non-coding RNAs (ncRNAs) that lead to increased biological complexity. By virtue of their ability to modulate, the flow of information from DNA to RNA to protein, ncRNAs have gained unprecedented attention (Morris and Mattick, 2014). Recent advancement in the next generation sequencing (NGS) technology has also allowed discovery of several classes of ncRNAs. IncRNAs, defined by convention as longer than, 200 nucleotides, form the largest class of ncRNAs. 
lncRNAs are involved in a variety of functions in the nervous system, by targeting multiple processes through regulation of genes and proteins. The lncRNA may be an antisense to a messenger RNA (mRNA) thus regulating its stability or translation. Alternatively, they may recruit protein factors on the gene promoter or on the mRNA, switching on or off expression from the locus. They may also drive localization of the mRNA to specific sub-cellular compartments. lncRNA can also exert epigenetic effects by recruiting chromatin modifiers to target gene loci. Presence of lncRNAs in multi-protein complexes suggests that they can also be a scaffold for binding of other RNA and protein components (Batista and Chang, 2013).

A remarkably large number of ncRNAs especially microRNAs (miRNAs) of 19 to 24 nucleotide length are present in dendrites leading to the suggestion that they are "pervasively" dendritic (Smalheiser, 2014). Many ncRNAs act through their membership in ribonucleoprotein complexes. In keeping with the view that ncRNAs play a key function in dendrites, several RNA binding proteins are also localized to the same sub-cellular location (Weiler et al., 1997). These include RNAi pathway proteins, members of the splicing machinery and miRNA biogenesis proteins. There is ample evidence suggesting irreplaceable role of miRNAs in dendritogenesis, spine morphogenesis, maturation, synaptic development and/or plasticity (Reviewed in detail Iyengar et al., 2014; Schratt, 2009; Smalheiser and Lugli, 2009; Weiß et al, 2015; Ye et al., 2016). Certain specific miRNAs, for instance, miR-134, has been found in the dendritic spines of cultured hippocampal neurons (Schratt et al., 2006). It appears that these miRNAs are engaged in activity dependent, local modulation of translation that can in turn lead to stimulus dependent remodeling of dendritic spines. It is becoming increasingly obvious that many steps of gene expression, especially miRNA biogenesis and splicing, conventionally thought to be performed in the nucleus also occur in the dendritic spine. The presence of mRNAs that contain retained introns and precursors of miRNAs in the dendritic spines (Lugli et al., 2008) suggest that RNA is held in an inactive state which can be rapidly processed following external stimuli. Apart from miRNAs, IncRNA being multi-functional molecules have emerged as important components of specialized neuronal compartments. Here, we describe four lncRNAs that are known to modulate neuronal architecture (Table 1).

In the pre-NGS era, BC1 RNA was identified by in situ hybridization of RNA probes in the dendrites of rat neurons (Tiedge et al., 1991). This rodent specific $B C 1$ RNA and its primate specific functional analog $B C 200$ is a typical RNA polymerase III transcribed gene almost exclusively expressed in nervous system. These cytoplasmic RNAs are present in the somatic and dendritic compartments of the central and peripheral nervous system (Tiedge et al., 1993, 1991). Expression of BC1 RNA coincides with the functional maturation of dendrites and synaptogenesis in hippocampal neurons, which can be further modulated by electrical activity of neurons (Muslimov et al., 1998). BC1 RNA appears to be a part of a high molecular mass ribonucleoprotein complex in neurons. Although these RNAs do not show strong sequence conservation, their specific location in post-synaptic compartments of rodent and primate neurons may indicate conserved functions related to pre- or post-translational events (Martignetti and Brosius, 1995; Tiedge et al., 1993). An attempt to study the function of $B C l$ RNA using knockout

Table 1: Summary of IncRNAs modulating neuronal architecture

\begin{tabular}{|c|c|c|c|c|}
\hline ncRNA & Functions & Interacting partners & Associate disease & Reference \\
\hline $\mathrm{BC} 1, \mathrm{BC} 200$ & $\begin{array}{l}\text { Part of ribonucleoprotein complex, } \\
\text { modulate exploration and anxiety } \\
\text { related behaviour }\end{array}$ & $\begin{array}{l}\text { FMRP, Pur } \alpha \text { and } \\
\beta, \text { SRP9/14, } \\
\text { HnRNP A2, PABP }\end{array}$ & Alzheimer's disease & $\begin{array}{l}\text { (Sosiñska } \text { et al., 2015; } \\
\text { Smalheiser, 2014; } \\
\text { Tiedge } \text { et al., 1991, 1993) }\end{array}$ \\
\hline Malat1 & Synaptogenesis & miR-124 & Parkinson's disease & $\begin{array}{l}\text { (Bernard et al., 2010; Liu et al., } \\
\text { 2017) }\end{array}$ \\
\hline $7 \mathrm{H} 4$ & $\begin{array}{l}\text { Precursor of miR-206 and miR-133b } \\
\text { at dendrites }\end{array}$ & Not yet known & Not yet known & $\begin{array}{l}\text { (Rodriguez et al., 2004; Valdez } \\
\text { et al., 2014) }\end{array}$ \\
\hline Durga & $\begin{array}{l}\text { Modulator of dendritic length and } \\
\text { number }\end{array}$ & Not yet known & Not yet known & (Sarangdhar et al., 2017) \\
\hline
\end{tabular}


(KO) mice revealed no visible change in brain morphology. However, the KO mice showed subtle behavioral changes such as reduced exploration and increased anxiety without change in spatial memory (Lewejohann et al., 2004). At the molecular level, the knockout of $B C 1$ alters efficiency of D2-mediated transmission but not the sensitivity of cannabinoid CB1 receptors. This specific effect is mediated by increase in Dopamine D2 receptor (D2DR) protein levels in the striatum of $\mathrm{BC} 1-\mathrm{KO}$ mice affecting the functional pool of receptors (Centonze et al., 2007).

Another lncRNA abundantly expressed in neurons is Metastasis-associated lung adenocarcinoma transcript 1, Malat1 (Neat2). In cultured hippocampal neurons, loss of this lncRNA has been shown to decrease synaptic density. Gene expression analysis after knockdown of Malat1 suggests its role in synapse formation in neurons, executed by regulating expression of genes involved in dendrite and synapse development(Bernard et al., 2010). Interestingly, Malat1 has several miRNA binding sites, for example, Malat1 directly interacts with and mutually represses brain enriched miR-124. The expression of Malatl is increased while miR124 is decreased in a model of Parkinson's disease (PD), a neurodegenerative disorder characterized by loss of dopaminergic neurons (DA) and motor control with cognitive deficits (Liu et al., 2017). In an attempt to identify differentiation regulating lncRNAs, Malat 1 found to be significantly induced in a model of in vitro differentiation of mouse neuroblastoma (N2a) cells by serum withdrawal and Retinoic Acid treatment. Loss of Malat1 in this model led to the inhibition of MAPK/ERK signalling pathway and reduction in average neurite length (Chen et al., 2016).

Apart from regulation of gene expression at cell body of neurons, IncRNAs can act as precursor or sponge for miRNAs at synapse. For example, synapse-associated lncRNA $7 H 4$ have precursor sequence for miR-206 and miR-133b embedded within its transcript. 7H4 RNA was identified as an RNA enriched at the motor endplate of skeletal neuromuscular junction (NMJ) (Velleca et al., 1994). Interestingly, like $7 H 4$, miR-206 and miR-133b are also enriched in the synapse and gets up-regulated at postnatal stages simultaneously, hinting at the possibility of active transport and local processing of long non-coding miRNA host transcripts to sub-cellular compartments away from the nucleus (Rodriguez et al., 2004; Valdez et al., 2014). Such local processing of host lncRNAs in specialized compartments may protect processed transcript products from unwanted interactions.

Recently, we discovered a lncRNA that regulates dendritogenesis during development in zebrafish. This IncRNA partially overlaps with the 5' end of the Kalirin gene (Sarangdhar et al., 2017), a RhoGEF kinase present in dendrites and synapse of neurons. Ectopic expression of this lncRNA by injection in the single cell stage of zebrafish embryos led to an increase in Kalirin mRNA expression (Fig. 2) (Sarangdhar et al., 2017).

Kalirin, an essential player in dendritic outgrowth and branching derives its curious name from its multidomain nature resembling the multi handedness of the Indian goddess Kali (Alam et al., 1997, Yan, 2015). IncRNA antisense to Kalirin was named Durga after the Indian goddess who is presented in mythology as the alter ego of "Kali" (Sarangdhar et al., 2017). Cultured neurons derived from the 48 hour old

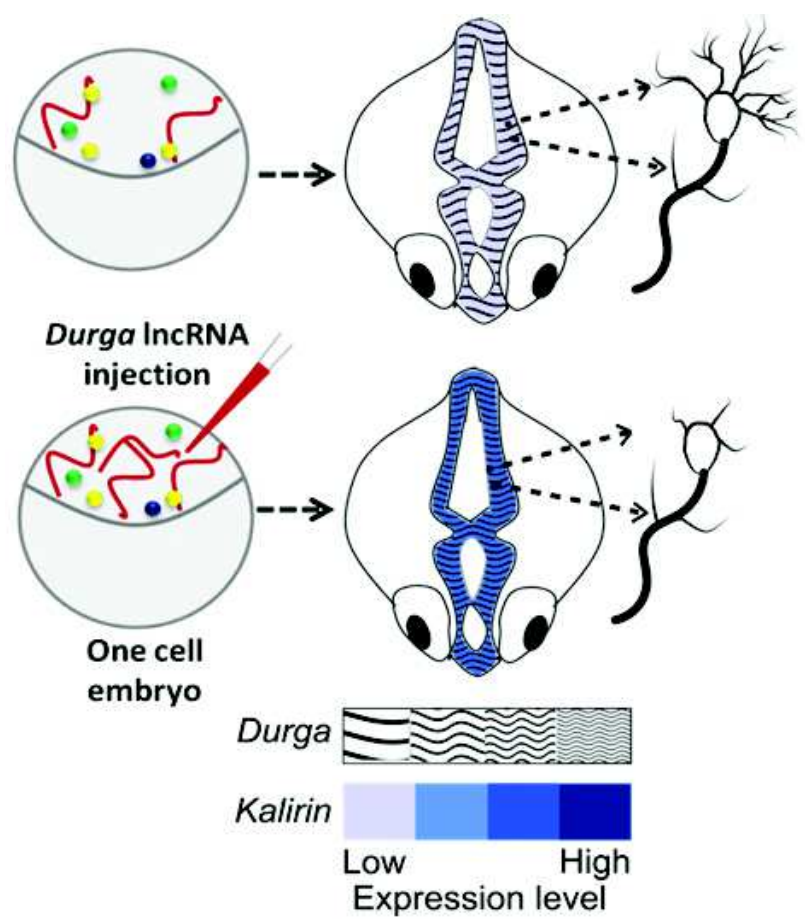

Fig. 2: Schematic of Durga IncRNA action. Ectopic overexpression of Durga IncRNA by microinjection in one cell stage zebrafish embryos increase Kalirin RNA expression at $24 \mathrm{hpf}$ and decrease dendritic density and length in neuronal culture 
zebrafish embryos which received Durga transcripts by microinjection at the single celled stage showed a severe reduction in the number of dendrites (Sarangdhar et al., 2017). Using total ribosome fractionation, we see that Durga over-expression results in increased localization of Kalirin transcript to the ribosomes fraction (Beena Pillai, unpublished observation). This implies, lncRNA Durga may localize Kalirin mRNA to the ribosome and protect it from degradation factors. Further investigation is required to find possible interactions of Durga $\operatorname{lncRNA}$ with potential mRNA stability or degradation factors. Although lncRNAs are poorly conserved, the human and mouse genomes also harbor an lncRNA at the 5' end of the Kalirin gene (Sarangdhar et al., 2017).

Looking at the close association of Kalirin with PSD95 in the post synaptic density (Xie et al., 2007) and role of Kalirin in microfilament re-organization of dendritic spines, the ability of Durga to modulate local Kalirin translation needs to be investigated with respect to neuronal activity. Further, differential regulation of Kalirin transcript isoforms in the developing brain and the potential involvement of Durga in this process are exciting prospects. Lastly, the relevance of the syntenically conserved lncRNA in the mammalian genome will be revealed through studies in the primary neurons of mouse which also provide suitable models for in vitro dendritogenesis.

\section{Conclusion and Future Perspectives}

The current understanding of lncRNAs in dendritogenesis, axogensis, synaptogenesis and spine modulation is in its infancy. However, looking at the multi-functional nature of lncRNAs and evidence of a few lncRNAs localized in specialized compartments of neurons promises widespread role in advanced functions of the brain such as memory and cognition. Further, the loss of function of these lncRNAs is also likely to be linked to the neuropsychiatric and neurodegenerative disorders because of the inherent role of dendritogenesis, axogensis, synaptogenesis and spine modulation in such pathological conditions.

In future, studies mayfocus on presence of lncRNAs in synaptic vesicles and neuronal exosomes that have received little attention and remain poorly

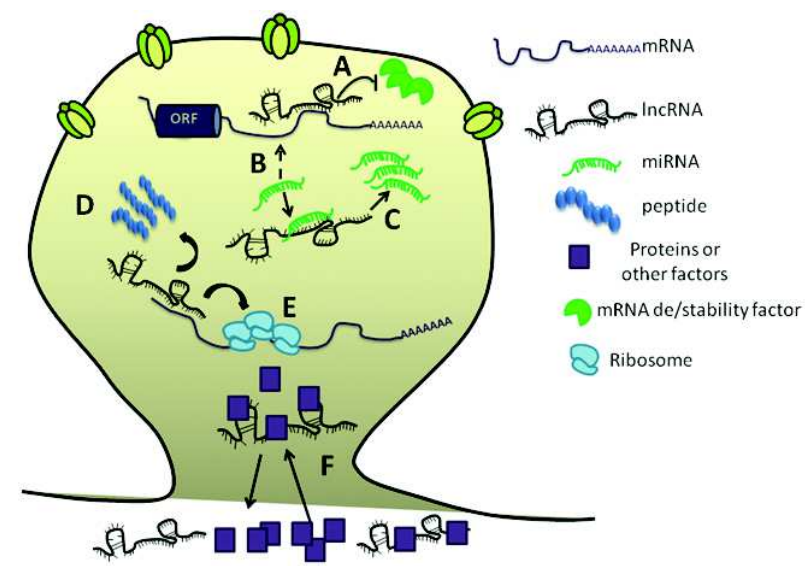

Fig. 3: Schematic of proposed mechanisms of action of IncRNAs in dendritic spine. A) Control of RNA half life by modulating its interactions with stability or destability factors by IncRNA B) Scavenging of miRNAs by IncRNA to de-repress miRNA targets C) IncRNAs as a source of miRNAs D) IncRNAs as a source of small peptides E) Control of translation by modulating recruitment of ribosomes on RNA F) Transport of factors in and out of the dendrites by IncRNAs

understood. How lncRNAs contribute to synaptic plasticity in the mammalian brain is still an open question (Fig. 3). The physiological and pathological functions of synaptic lncRNAs need to be explored further. The classification of lncRNAs based on their localization in specialized neuronal compartments is under-explored perhaps because of continuity of cytoplasm of neuronal cell body and axons/dendrites, sometimes blurring their boundaries. Transfer of synaptic lncRNAs from the synapse to the nucleus and vice versa to participate in the signaling has not yet been demonstrated. The innate potential of IncRNAs to be transported as cargo during vesicular transfer and their ability to act by sponging miRNAs or scaffolding protein factors in the central nervous system is not explored.

We hope that detailed investigations on questions posed above will reveal the importance of IncRNA in Cajal's century old neuroscience puzzle. Sculpting of neurons in general and more specifically, the role of novel lncRNAs like Durga in this dynamic process that underlies learning and memory promise an exciting period of discovery. 


\section{References}

Alam M R, Johnson R C, Darlington D N, Hand T A, Mains R E, Eipper B A (1997) Kalirin, a cytosolic protein with spectrinlike and GDP/GTP exchange factor-like domains that interacts with peptidylglycine alpha-amidating monooxygenase, an integral membrane peptide-processing enzyme J Biol Chem 272: 12667-12675

Batista P J and Chang H Y (2013) Long Noncoding RNAs: Cellular Address Codes in Development and Disease Cell 152 12981307

Bernard D, Prasanth K V, Tripathi V, Colasse S, Nakamura T, Xuan Z, Zhang M Q, Sedel F, Jourdren L, Coulpier F, et al. (2010) A long nuclear-retained non-coding RNA regulates synaptogenesis by modulating gene expression $E M B O \mathrm{~J}$ 29 3082-3093

Bramham C R and Wells D G (2007) Dendritic mRNA: Transport, translation and function Nat Rev Neurosci 8 776-789

Centonze D, Rossi S, Napoli I, Mercaldo V, Lacoux C, Ferrari F, Ciotti M T, De Chiara V, Prosperetti C, Maccarrone M, et al. (2007) The Brain Cytoplasmic RNA BC1 Regulates Dopamine D2 Receptor-Mediated Transmission in the Striatum J Neurosci 27 8885-8892

Chen L, Feng P, Zhu X, He S, Duan J and Zhou D (2016) Long non-coding RNA Malat1 promotes neurite outgrowth through activation of ERK/MAPK signalling pathway in N2a cells J Cell Mol Med 20 2102-2110

Graveland GA, Williams R S and DiFiglia M (1985) Evidence for degenerative and regenerative changes in neostriatal spiny neurons in Huntington's disease Science 227 770-3

Hu X, Ballo L, Pietila L, Viesselmann C, Ballweg J, Lumbard D, Stevenson M, Merriam E and Dent E W (2011) BDNFInduced Increase of PSD-95 in Dendritic Spines Requires Dynamic Microtubule Invasions J Neurosci 31 1559715603

Iyengar B R, Choudhary A, Sarangdhar M A, Venkatesh K V, Gadgil C J and Pillai B (2014) Non-coding RNA interact to regulate neuronal development and function Front Cell Neurosci 847

Jan Y-N and Jan L Y (2010) Branching out: mechanisms of dendritic arborization Nat Rev Neurosci 11 316-328

Kandel E R, Schwartz J H and Jessell T M (2013) Principles of Neural Science

Kovalchuk Y, Eilers J, Lisman Jand Konnerth A (2000) NMDA receptor-mediated subthreshold $\mathrm{Ca}(2+)$ signals in spines of hippocampal neurons J Neurosci 20 1791-1799

Kweon J H, Kim S and Lee S B (2017) The cellular basis of dendrite pathology in neurodegenerative diseases BMB Rep
50 5-11

Lewejohann L, Skryabin B V, Sachser N, Prehn C, Heiduschka P, Thanos S, Jordan U, Dell'Omo G, Vyssotski A L, Pleskacheva M G et al. (2004) Role of a neuronal small non-messenger RNA: behavioural alterations in BC1 RNAdeleted mice Behav Brain Res 154 273-289

Liu W, Zhang Q, Zhang J, Pan W, Zhao J and Xu Y (2017) Long non-coding RNA MALAT1 contributes to cell apoptosis by sponging miR-124 in Parkinson disease Cell Biosci 7 19

Lugli G, Torvik V I, Larson J and Smalheiser N R (2008) Expression of microRNAs and their precursors in synaptic fractions of adult mouse forebrain J Neurochem 106 650-661

Martignetti J A and Brosius J (1995) BC1 RNA: transcriptional analysis of a neural cell-specific RNA polymerase III transcript Mol Cell Biol 15 1642-1650

Moroz L L (2011) Aplysia Curr Biol 21 60-61

Morris K V and Mattick J S (2014) The rise of regulatory RNA Nat Rev Genet 15 423-437

Muslimov IA, Banker G, Brosius J and Tiedge H (1998) Activitydependent Regulation of Dendritic BC1 RNA in Hippocampal Neurons in Culture J Cell Biol 1411601 LP-1611

Nimchinsky E A, Sabatini B L and Svoboda K (2002) Structure and Function of Dendritic Spines Annu Rev Physiol 64 313-353

Okabe S (2007) Molecular anatomy of the postsynaptic density Mol Cell Neurosci 34 503-518

Penzes P, Cahill M E, Jones K A, VanLeeuwen J-E and Woolfrey K M (2011) Dendritic spine pathology in neuropsychiatric disorders Nat Neurosci 14 285-293

Pitman R M (1984) The versatile synapse J Exp Biol 112 199224

Ramon y Cajal S (1906) The structure and connexions of neurons Nobel Lect Physiol or Med 1901-1921 220-253

Ramos A D, Andersen R E, Liu S J, Nowakowski T J, Hong S J, Gertz C C, Salinas R D, Zarabi H, Kriegstein A R and Lim D A (2015) The long noncoding RNA Pnky regulates neuronal differentiation of embryonic and postnatal neural stem cells Cell Stem Cell 16 439-447

Riccomagno M M and Kolodkin A L (2015) Sculpting Neural Circuits by Axon and Dendrite Pruning Annu Rev Cell Dev Biol 31 779-805

Rodriguez A, Griffiths-Jones S, Ashurst J L and Bradley A (2004) Identification of Mammalian microRNA Host Genes and Transcription Units Genome Res 14 1902-1910

Sarangdhar M A, Chaubey D, Bhatt A, K M M, Kumar M, 
Ranjan S and Pillai B (2017) A Novel Long Non-coding RNA, durga Modulates Dendrite Density and Expression of kalirin in Zebrafish Front Mol Neurosci 10 1-10

Schratt G (2009) MicroRNAs at the synapse Nat Rev Neurosci $10842-849$

Schratt G M, Tuebing F, Nigh E A, Kane C G, Sabatini M E, Kiebler M and Greenberg M E (2006) Corrigendum: A brain-specific microRNA regulates dendritic spine development Nature 441 902-902

Šišková Z, Justus D, Kaneko H, Friedrichs D, Henneberg N, Beutel T, Pitsch J, Schoch S, Becker A, vonderKammer H, et al. (2014) Dendritic structural degeneration is functionally linked to cellular hyperexcitability in a mouse model of alzheimer's disease Neuron 84 1023-1033

Smalheiser N R (2014) The RNA-centred view of the synapse: Non-coding RNAs and synaptic plasticity Philos Trans $R$ Soc B Biol Sci 369 20130504-20130504

Smalheiser N R and Lugli G (2009) microRNA Regulation of Synaptic Plasticity Neuro Molecular Med 11 133-140

Sosiñska P, Mikuª-Pietrasik J and Ksiazek K (2015) The doubleedged sword of long non-coding RNA: The role of human brain-specific BC200 RNA in translational control, neurodegenerative diseases, and cancer Mutat Res - Rev Mutat Res 766 58-67

Sotrel A, Williams R S, Kaufmann W E and Myers R H (1993) Evidence for neuronal degeneration and dendritic plasticity in cortical pyramidal neurons of Huntington's disease: A quantitative Golgi study Neurology 43 2088-2096

Tiedge H, Chen W and Brosius J (1993) Primary structure, neuralspecific expression, and dendritic location of human BC200
RNA J Neurosci 132382 LP-2390

Tiedge H, Fremeau R T, Weinstock P H, Arancio O and Brosius J (1991) Dendritic location of neural BC1 RNA Proc Natl Acad Sci U S A 88 2093-2097

Valdez G, Heyer M P, Feng G and Sanes J R (2014) The Role of Muscle microRNAs in Repairing the Neuromuscular Junction PLoS One 9 e93140

Velleca MA, Wallace M C and Merlie J P (1994) A Novel SynapseAssociated Noncoding RNA Mol Cell Biol 7095-7104

Vetter P, Roth A and Häusser M (2001) Propagation of action potentials in dendrites depends on dendritic morphology J Neurophysiol 85 926-937

Weiler I J, Irwin S a, Klintsova a Y, Spencer C M, Brazelton a D, Miyashiro K, Comery T a, Patel B, Eberwine J and Greenough W T (1997) Fragile X mental retardation protein is translated near synapses in response to neurotransmitter activation Proc Natl Acad Sci U S A 94 5395-5400

Weiß K, Antoniou A and Schratt G (2015) Non-coding mechanisms of local mRNA translation in neuronal dendrites Eur J Cell Biol 94 363-367

Wu P, Zuo X, Deng H, Liu X, Liu L, Ji A., 2013. Roles of long noncoding RNAs in brain development, functional diversification and neurodegenerative diseases Brain Res Bull 97 69-80

Xie Z, Srivastava D P, Photowala H, Kai L, Cahill M E, Woolfrey K M, Shum C Y, Surmeier D J and Penzes P(2007) Kalirin7 Controls Activity-Dependent Structural and Functional Plasticity of Dendritic Spines Neuron 56 640-656

Ye Y, Xu H, Su X and He X (2016) Role of MicroRNA in Governing Synaptic Plasticity Neural Plast 2016. 